\title{
Comparison of wheel-rail contact modelling in multibody system online simulation
}

\author{
Binbin Liu ${ }^{1[0000-0003-2482-8729]}$, Stefano Bruni ${ }^{2[0000-0003-2177-5254]}$ \\ 1,2 Dipartimento di Meccanica, Politecnico di Milano, Via La Masa 1, Milano 20156, Italy \\ binbin.liuepolimi.it
}

\begin{abstract}
The wheel-rail contact modelling is always an interesting topic in rail vehicle system dynamics simulation. Many contact models have been developed for different purposes, and each model has its own pros and cons for different applications. In multibody system (MBS) simulation of rail vehicles, the efficiency and accuracy of the wheel-rail contact model are of importance. It is the aim of this paper to compare in MBS online simulation one classical approach (Hertz theory+FASTSIM), one approximated non-Hertzian approach and the 'exact' solver CONTACT and show the influences of the contact modelling on the results of vehicle dynamics simulations.
\end{abstract}

Keywords: wheel-rail contact, vehicle dynamics, contact modelling, nonHertzian contact.

\section{Introduction}

The wheel-rail contact modelling is one of the most important aspects in multibody system (MBS) simulations of rail vehicles since it reflects the highly nonlinear characteristics of the system. Many contact models have been developed for different purposes in railway applications [1].

Generally, the wheel-rail contact problem is assumed to be decoupled in the normal and tangential direction. Therefore, the two components can be treated separately which results in varied combinations of contact solvers. The Manchester Contact Benchmark was defined [2] to compare contact models commonly used in rail vehicle dynamics simulations. This benchmark was designed Case A and Case B. However, only the results concerning Case A were published, which show the comparisons in the context of offline (post-processing) and focus on the local contact solution rather than the influence of the contact modelling on the online dynamics of the vehicles which are basically the task of Case B. In addition, it is worth mentioning that a comprehensive investigation on the accuracy of different simplified tangential contact algorithms has been performed with reference to CONTACT for Hertzian contacts in [3].

To gain a complete insight into the wheel-rail contact phenomena and relevant issues such as the train-track interaction, degradation of the wheel-rail interface and infrastructure, the integration of an advanced contact model in MBS is required. 
Recently, Burgelman et al. [4] studied the influence of wheel-rail contact modelling on vehicle dynamic simulation by means of co-simulation between commercial software VI-Rail and Matlab. However, these external contact models were actually not incorporated into VI-Rail directly instead a correction procedure was introduced to compensate the difference caused by the default contact model. Moreover, the difference of the contact solution considered in this work is mainly due to the variation in the normal contact solver.

In contrast, the aim of this work is to investigate the influence of wheel-rail contact modelling in the MBS online simulation of rail vehicles. To this end, a methodology is proposed to integrate external contact models including both normal and tangential contact solvers into the MBS vehicle-track interaction model. The contact model combinations considered in this work represent three levels of elaboration of modelling, namely the classic Hertz+FASTSIM [5] that is the built-in solver of SIMPACK for wheel-rail contact problem, Kik-Piotrowski (KP) [6] method to solve the normal problem and Kalker book of tables non-Hertzian $(\mathrm{KBTNH})[7,8]$ to solve the tangential problem which is an advanced approximate non-Hertzian contact solver and the well-known 'exact' solver CONTACT [9]. The Manchester benchmark vehicle [10] was selected as a baseline to study the dynamics of the vehicle considering the various contact models. The tangential and normal contact solutions are reported and compared.

\section{$2 \quad$ MBS model}

The simulation code SIMPACK Rail is used for building the MBS model of a rail vehicle which is a passenger vehicle model adapted based on the Manchester benchmark model as shown in Fig. 1. The MBS vehicle model consists of a carbody, two bogies with two stages of suspensions and four solid axle wheelsets. The model is made up of rigid bodies with 46 degrees of freedom. The primary and secondary suspensions are modelled with linear spring and damping elements. The lateral displacement between carbody and bogie frame is limited by lateral bump stops, and the relative movements are damped by the dampers arranged in the vertical and lateral directions. The main parameters of the Manchester benchmark model are summarized in Table 1.

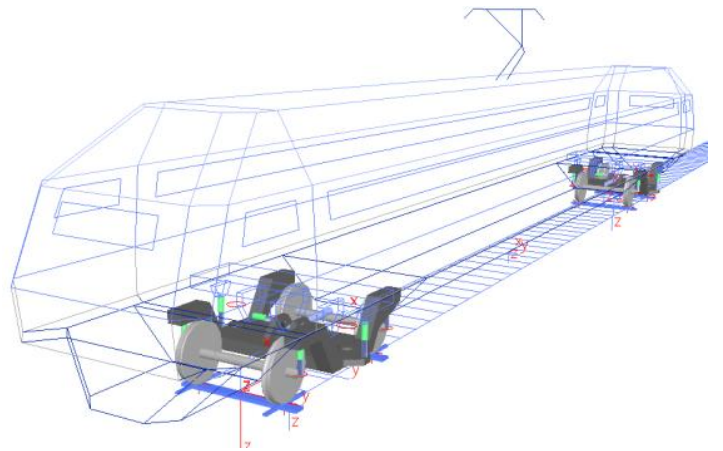

Fig. 1. MBS vehicle model. 
Table 1. Main parameters of the vehicle-track system based on Manchester benchmark.

\begin{tabular}{lll}
\hline Element & Value & Unit \\
\hline Carbody mass & 32000 & $\mathrm{~kg}$ \\
Bogie frame mass & 2615 & $\mathrm{~kg}$ \\
Wheelset mass & 1813 & $\mathrm{~kg}$ \\
Primary longitudinal stiffness & 31391 & $\mathrm{kN} / \mathrm{m}$ \\
Primary longitudinal damping & 15.0 & $\mathrm{kNs} / \mathrm{m}$ \\
Primary lateral stiffness & 3884 & $\mathrm{kN} / \mathrm{m}$ \\
Primary lateral damping & 2.0 & $\mathrm{kNs} / \mathrm{m}$ \\
Track gauge & 1435 & $\mathrm{~mm}$ \\
Track segment mass & 330 & $\mathrm{~kg}$ \\
Lateral stiffness of the sleeper for a single rail & 20000 & $\mathrm{kN} / \mathrm{m}$ \\
Vertical stiffness of the sleeper for a single rail & 75000 & $\mathrm{kN} / \mathrm{m}$ \\
Wheel radius & 460 & $\mathrm{~mm}$ \\
Friction coefficient & 0.4 & - \\
Wheel/rail profile combination & $\mathrm{S} 1002 / \mathrm{UIC6} 60$ & - \\
Rail inclination & $1: 20$ & - \\
\hline
\end{tabular}

The resultant normal contact force is determined by kinematic constraint [11] which is rigorous for a single rigid contact between a pair of wheel and rail [12]. Therefore, the wheel/rail profile combination S1002/UIC60 with rail inclination 1:20 was selected to ensure a single point of contact between the wheel and rail for the simulation condition considered in this study. The contact point distribution computed with SIMPACK is shown in Fig. 2. The tangential contact problem is solved with the FASTSIM algorithm, which is switched off when an external tangential contact model is activated. The track flexibility is considered by introducing a moving ballast sectional model of the track with lateral and vertical elasticities under each wheelset [13].

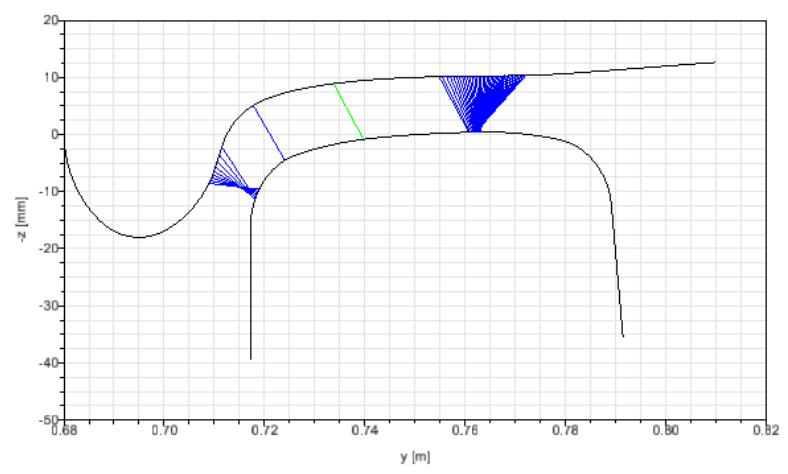

Fig. 2. Contact point distribution for combination of S1002/UIC60 (1:20) 


\section{Integration of contact models into MBS}

The following combinations of contact models were implemented in this work as examples and apparently this methodology can be easily extended to any combinations of contact model.

Hertz+FASTSIM: This combination is the default option in SIMPACK Rail module, where the Hertz theory is applied to solve the normal contact problem with an analytical form for elliptic contact patches and the FASTSIM algorithm is used to calculate the creep forces. It should be mentioned that the contribution of the moment produced in the contact patch has not been considered in this option since this contribution is marginal.

KP+KBTNH: This model applies KP method to solve the normal contact problem, which is a fast and simplified non-Hertzian method based on the concept of virtual penetration, for more details the reader is refer to reference [6]. The KBTNH model is used to calculate the creep forces and moment [7, 8]. This model is coded in Matlab and exchanges data with the MBS vehicle model via co-simulation.

CONTACT: The program CONTACT is used for solving both normal and tangential contact problem with algorithms of NormCG and TangCG, respectively [14]. In order to incorporate CONTACT into SIMPACK for online simulation, a multi-co-simulation platform is established among CONTACT, SIMPACK and Matlab/Simulink.

The idea of implementing the co-simulations is to determine the resultant normal force, contact position on the wheel and rail, contact angle and creepages in SIMPACK with the MBS vehicle model, which are fed to the external contact models as inputs, then the creep forces and moment calculated by the external contact models are fed back to the MBS vehicle model to form a close loop. The schematic presentation of the proposed methodology is illustrated in Fig. 3.

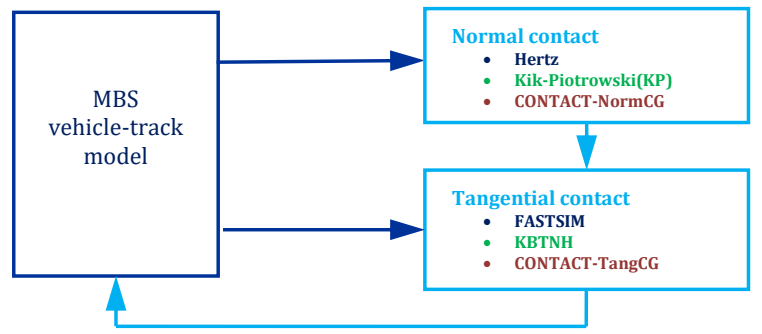

Fig. 3. Scheme of the integration of contact models into MBS simulation.

\section{$4 \quad$ Results and discussion}

In order to validate the proposed methodology, the FASTSIM algorithm is implemented in Matlab and integrated into the MBS model by means of the methodology introduced in Section 3. The simulation results are compared to those of the default FASTSIM in SIMPACK. The comparisons (results not shown here for the sake of conciseness) show that very good agreement has been achieved which proves the validity of the proposed 
methodology for the purpose of this work and ensures any differences obtain below purely due to the variation of contact models.

A case study considering the vehicle running on a tangent track at $80 \mathrm{~km} / \mathrm{h}$ with an initial lateral displacement of $4 \mathrm{~mm}$ in the two wheelsets of the leading bogie is presented in this section. The track irregularities are not considered to have a clear comparison of the results. The first $0.2 \mathrm{~s}$ of the time histories of the simulation results are cut off to remove the initial numerical peaks.

\subsection{Solution of tangential contact}

The results of the dynamics simulation of the vehicle in terms of the time histories of the creep forces and moment of the leading wheelset are compared in Fig. 4.
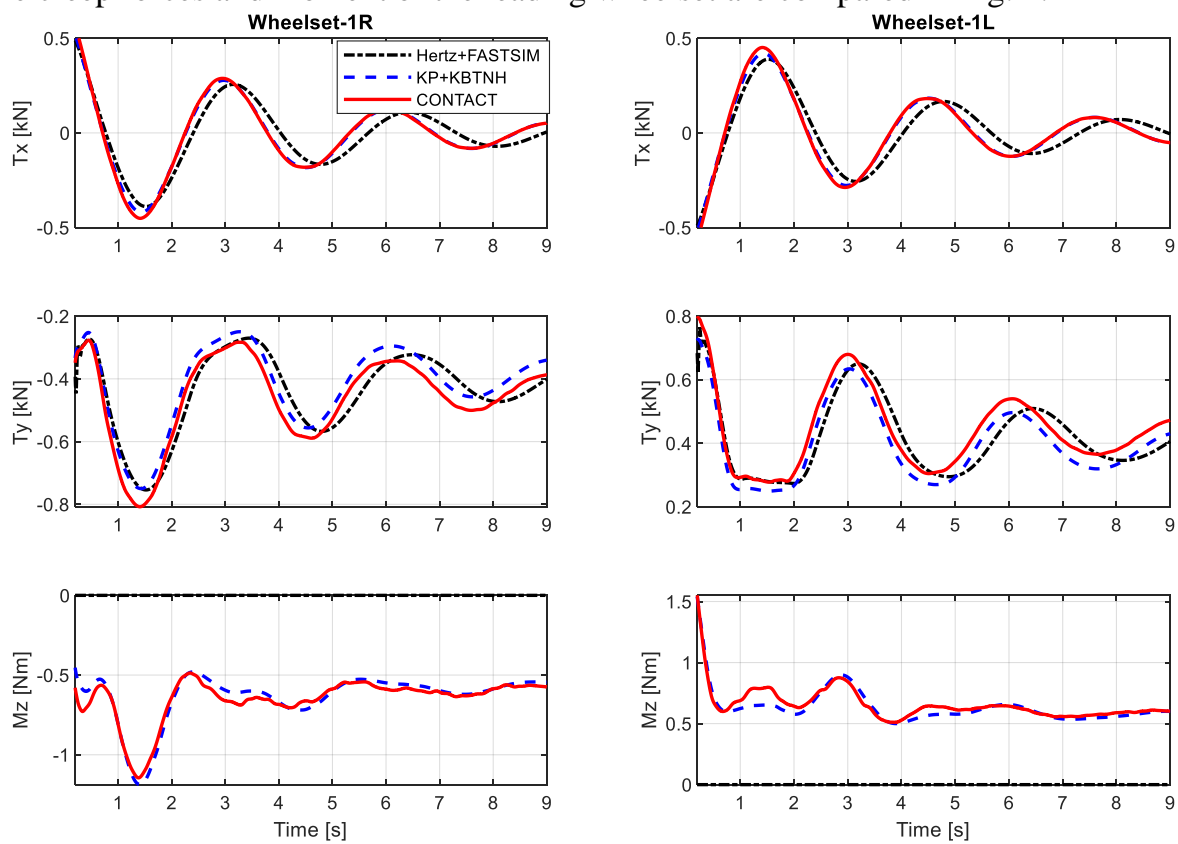

Fig. 4. Creep forces and moments of the wheelset 1.

Fig.4 shows the longitudinal creep force computed by a contact model, which includes a non-Hertzian solver for the normal contact problem, is greater than that of a contact model where the normal problem is solved by Hertzian model. This is probably due to the longitudinal creep force due to spin in the asymmetric contact patches (shown later in Fig.6) arises, while this effect is not considered in the Hertz model where the contact patch is assumed to be elliptic. The KP+KBTNH model shows good agreement to CONTACT for all the contact force components shown in Fig.4, especially the longitudinal creep forces which are almost identical to the results of CONTACT, whilst minor deviations are observed for the lateral creep forces. The magnitude of the moments is very small as expected. 
The differences of the results of Hertz+FASTSIM from those of the other two models are twofold since both normal and tangential contact solvers are different in different models. To better understand the contribution of the normal/tangential solver to the difference, more investigations shall be made with one variable at each time which will be part of our future work.

\subsection{Solution of normal contact}

The time histories of the normal contact solutions of the leading wheelset are presented in Fig.5. The contact shapes and the maximum pressure profile along the lateral axis at $1.5 \mathrm{~s}$ and $7.0 \mathrm{~s}$ are plotted in Fig.5.
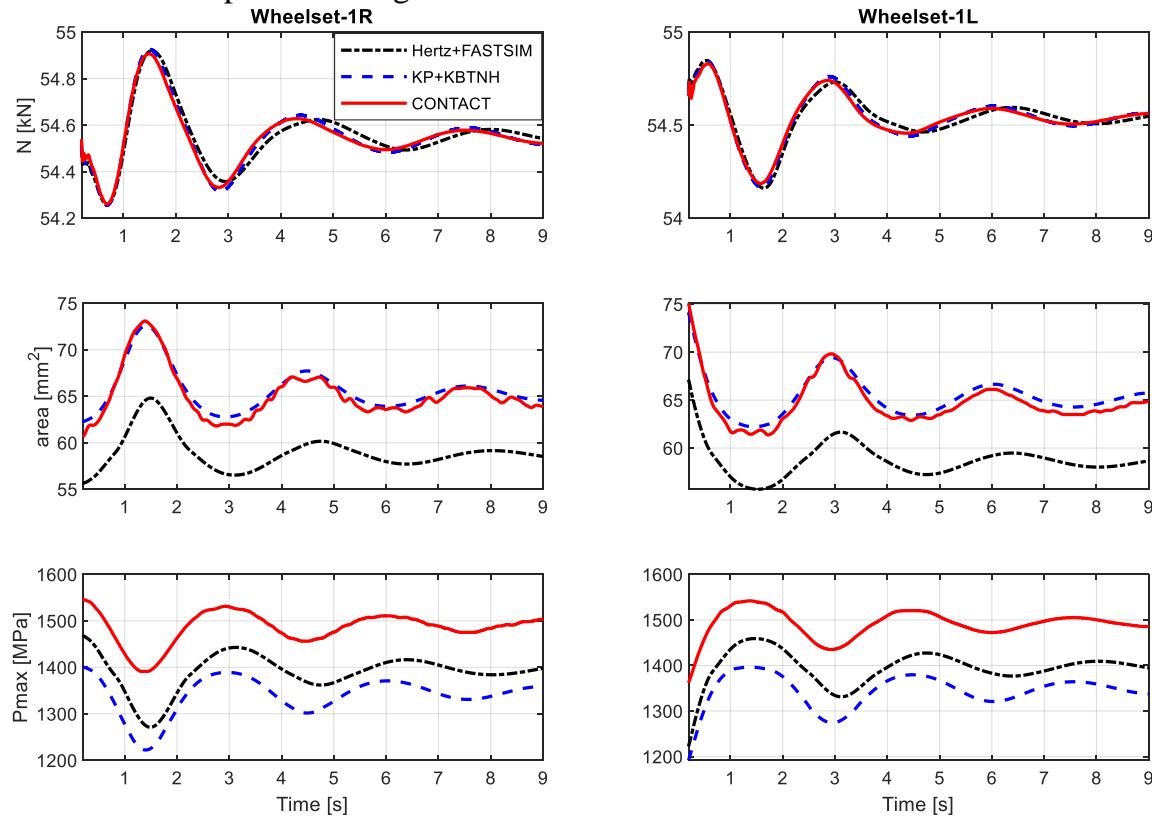

Fig. 5. Normal force, contact area and maximum contact pressure.

It can be seen from Fig. 5 that for the first $1.5 \mathrm{~s}$ the agreement in terms of the normal force is fairly good since the initial contact condition is the same for different contact models and the resultant normal force is obtained from MBS model. However, the difference increases with time up to approximately $3 \mathrm{~s}$, then remain in a relative stable level.

The time histories of the contact area obtained from KP+KBTNH and CONTACT are in good agreement, whereas the contact areas are underestimated by Hertz+FASTSIM approximately $12 \%$. For the maximum pressure, Hertz+FASTSIM underestimates it around 5\% while $\mathrm{KP}+\mathrm{KBTNH}$ under-estimates it about $9 \%$ with reference to CONTACT. These results confirm the findings in [15] that the KP model is able to predict the contact area 'correctly' with respect to CONTACT for most cases 
but fails to capture the summit of the pressure distribution for some non-elliptic contacts. Therefore, an improvement of this results can be expected with a more detailed normal contact model, e.g. Extended KP model [15].
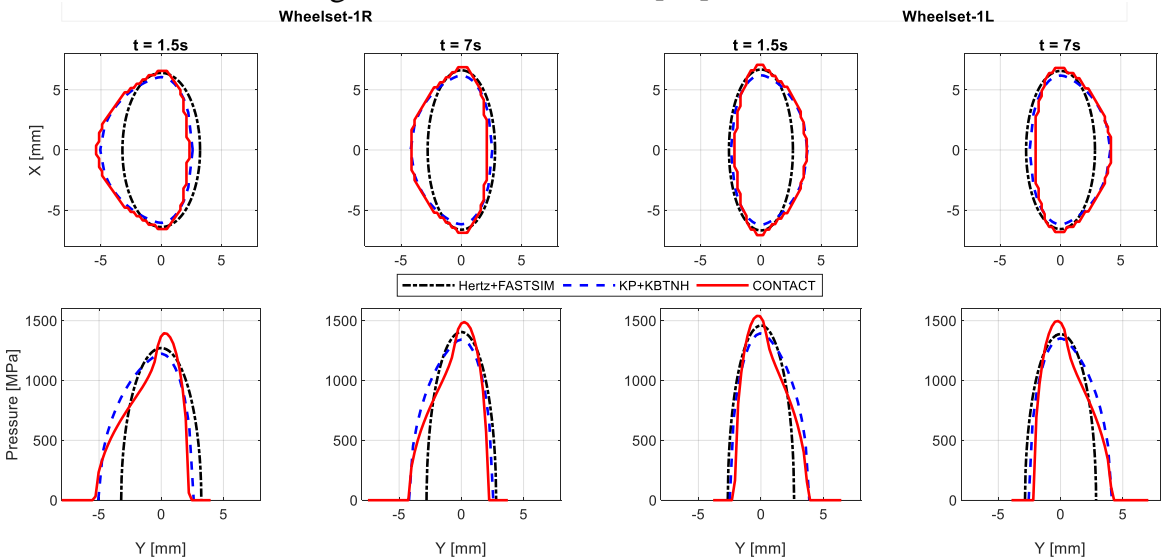

Fig. 6. Contact shape and maximum pressure profile along y axis.

Fig.6 show the solutions of the local contact analysis in terms of contact patch shape and distribution of normal pressures. Despite not being directly relevant to vehicle dynamics which is only affected by the resultant contact forces, the results of local contact analysis are crucial for contact damage analysis, e.g. wear and rolling contact fatigue. Observations from Fig.6 suggest that the contact shapes are SDEC [7] type which is the basic contact shape used in KBTNH method which also explain the good agreement of the creep forces between KP+KBTNH and CONTACT shown in Fig.4. The good agreement of the contact shape obtained from KP and CONTACT confirms the efficiency of the KP model in the determination of the contact shape. While the peak in the pressure is not well predicted by the KP method and this deviation contributes the difference in the lateral creep force shown in Fig.4. In conclusion, the KP+KBTNH method provides very similar results to CONTACT in terms of contact area and shape, however, the corresponding maximum pressure are underestimated. The same comments made on the contact area and pressure distribution as for the previous figure are still valid in this case.

\subsection{Lateral motion}

To investigate the influence of the differences in the contact solutions on the motion of the system, the lateral displacements of the wheelsets of the leading bogie, of the bogie and the carbody are reported in Fig.7-Fig 9, respectively. 

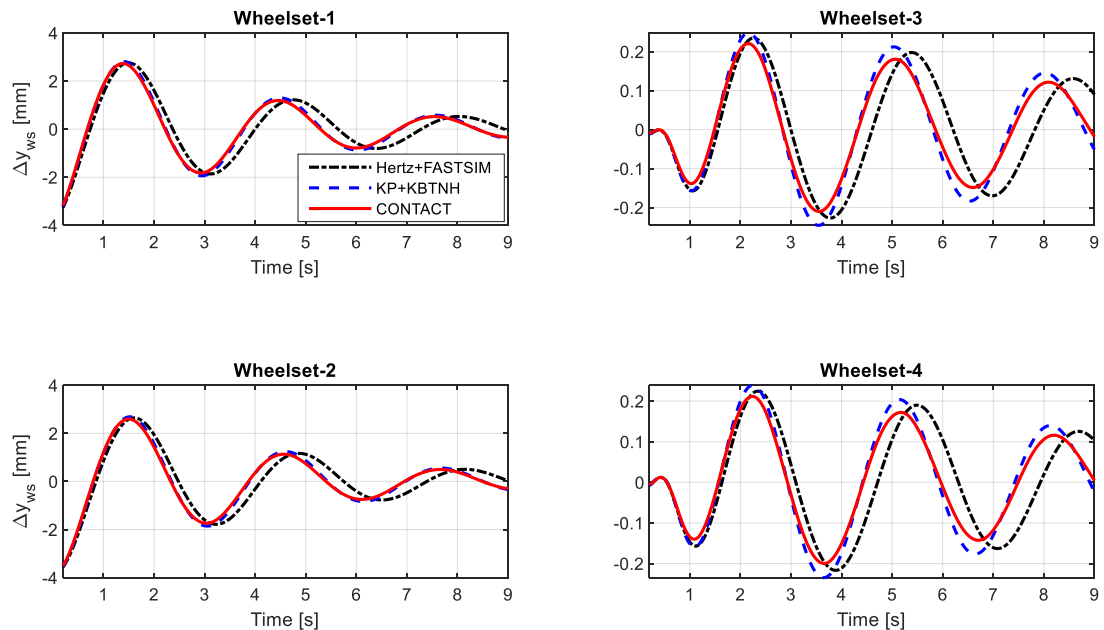

Fig. 7. Lateral displacement of wheelsets.

The lateral motions of the wheelsets obtained with different contact models show that the wavelength of the motion is longer when Hertzian model instead of the non-Hertzian contact model (KP+KBTNH and CONTACT) is applied. It means the more detailed contact model dissipates less energy during this motion. $\mathrm{KP}+\mathrm{KBTNH}$ is able to capture more realistic behavior of the system than classic model, considering the results obtained using CONTACT as a reference. It should also be noted that the scales of the plots for wheelsets 3 and 4 are different from those of the wheelsets 1 and 2, so the differences seem larger.

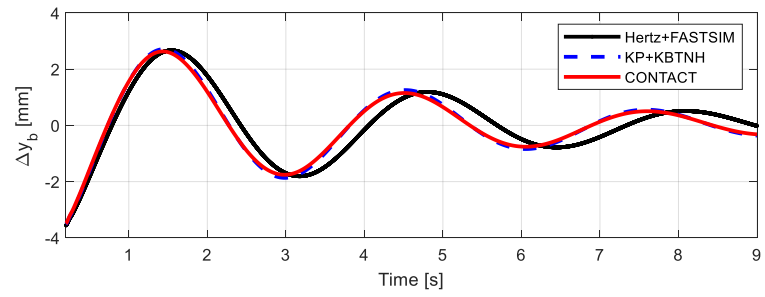

Fig. 8. Lateral displacement of the front bogie.

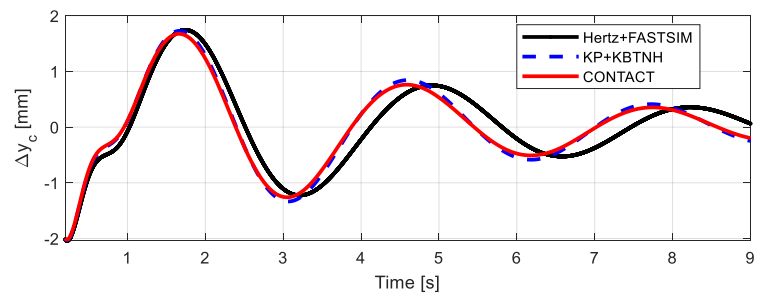

Fig. 9. Lateral displacement of the carbody. 
Due to the influence of contact modelling on the motion of the wheelset, the same trend is transmitted to the bogie and carbody via primary and secondary suspensions as shown in Fig. 8 and Fig.9 and again a better match is found between the results obtained using CONTACT and KP+KBTNH than with FASTSIM. These results confirm that the contact modelling is crucial for the MBS online simulation.

\section{Conclusions}

A methodology to implementing external contact models into MBS for online rail vehicle simulations is proposed. Based on the proposed methodology, three levels of elaboration of contact models are implemented in an MBS vehicle-track interaction system to investigate the influence of contact modelling in the MBS online simulation.

The simulation results obtained from the case study considered in this work show that the contact modelling influences wheel-rail contact parameters as well as vehicle dynamics. Future extension of this work is foreseen to investigate the effect of using different wheel-rail contact models on the estimation of stability and curving behavior.

The comparison of the efficiency of different models is also interesting, however, it is not the concern of the current work because the codes implemented are not yet optimized.

\section{References}

1. Sajjad Z. Meymand, Alexander Keylin \& Mehdi Ahmadian (2016) A survey of wheel-rail contact models for rail vehicles, Vehicle System Dynamics, 54:3, 386-428.

2. P. Shackleton and S. Iwnicki, Comparison of wheel-rail contact codes for railway vehicle simulation: An introduction to the Manchester Contact Benchmark and initial results, Veh. Syst. Dyn. 46 (2008), pp. 129-149.

3. Vollebregt E, Iwnicki S, Xie G, Shackleton P. Assessing the accuracy of different simplified frictional rolling contact algorithms. Veh Syst Dyn. 2012;50:1-17.

4. Nico Burgelman, Matin Sh. Sichani, Roger Enblom, Mats Berg, Zili Li \& Rolf Dollevoet (2015) Influence of wheel-rail contact modelling on vehicle dynamic simulation, Vehicle System Dynamics, 53:8, 1190-1203.

5. Kalker JJ. A fast algorithm for the simplified theory of rolling contact. Veh Syst Dyn. 1982;11:1-13.

6. W. Kik and J. Piotrowski. A fast, approximate method to calculate normal load at contact between wheel and rail and creep forces during rolling. In I. Zobory, editor, Proceedings of 2nd Mini-conference on contact mechanics and wear of rail/wheel systems, 1996.

7. Piotrowski, J., Liu, B., Bruni, S.,: The Kalker book of tables for non-Hertzian contact of wheel and rail. Vehicle System Dynamics 55(6), 875-901(2017).

8. J. Piotrowski, S. Bruni, B. Liu and Egidio: a fast method for determination of creep forces in non-Hertzian wheel-rail contact based on a book of tables, Multibody Syst Dyn (2018). https://doi.org/10.1007/s11044-018-09635-3.

9. J.J. Kalker: Three-dimensional elastic bodies in rolling contact. Kluwer Academic Publishers, 1990.

10. Iwnicki, S. (ed.): The Manchester Benchmarks for Rail Vehicle Simulation, Volume 31 of Supplement to Vehicle System Dynamics. Swets \& Zeitlinger, Lisse, 1999. 
11. Gunter Schupp, Christoph Weidemann and Lutz Mauer (2004) Modelling the Contact Between Wheel and Rail Within Multibody System Simulation, Vehicle System Dynamics, 41:5, 349-364.

12. Jean-Pierre Pascal, Jalil Rismantab Sany (2019): Dynamics of an isolated railway wheelset with conformal wheel-rail interactions, Vehicle System Dynamics, DOI: 10.1080/00423114.2018.155770.

13. Egidio Di Gialleonardo, Francesco Braghin, Stefano Bruni (2012): The influence of track modelling options on the simulation of rail vehicle dynamics, Journal of Sound and Vibration, 331(19): 4246-4258.

14. Vollebregt, E.A.H.,: User guide for CONTACT, Rolling and sliding contact with friction. Technical report TR09-03, version 17.1, VORtech BV, Delft, The Netherlands; 2017. Available from:www.kalkersoftware.org.

15. B. Liu, S. Bruni and E. Vollebregt: A non-Hertzian method for solving wheel-rail normal contact problem taking into account the effect of yaw, Vehicle System Dynamics, 2016, 54(9) 1226-1246. 